$63^{\text {ème }}$ Congrès de la SFCO, 03036 (2015)

DOI:10.1051/sfco/20156303036

(C) Owned by the authors, published by EDP Sciences, 2015

POSTER

\title{
Glossodynies : une manifestation inhabituelle du syndrome d'Eagle : $\underline{\text { à propos de deux cas }}$
}

\author{
Melka AC******, Depeyre A*, Pham Dang $\mathrm{N}^{* * * *}$, Barthélemy I**, Deschaumes $\mathrm{C}^{* * * * *}$ \\ * Clermont Université, Université d'Auvergne, Neuro-Dol, BP. 10448, 63000 Clermont-Ferrand \& Inserm \\ U1107, 63001 Clermont-Ferrand \\ ** CHU Clermont-Ferrand, Service de Chirurgie Maxillo-faciale, 63003 Clermont-Ferrand \\ *** CHU Clermont-Ferrand, Service d'Odontologie, 63003 Clermont-Ferrand
}

Le syndrome d'Eagle est caractérisé par une variété importante de symptômes non spécifiques qui rendent son diagnostic difficile. Il s'agit le plus souvent de sensation de corps étranger dans le pharynx, d'otalgie référées, de dysphagies et de douleurs cervicales augmentées lors de la rotation de la tête ou à l'ouverture de la bouche (Eagle 1949, Gossman 1977).

Observation

Deux patients rapportés, l'un de sexe masculin, 45 ans et l'autre de sexe féminin, 68 ans, ont été adressés au service d'Odontologie et au service de Chirurgie Maxillo-Faciale de Clermont Ferrand du CHRU de Clermont Ferrand suite à une errance diagnostique pour des glossodynies. Ils présentaient une sensation à type de brulure au niveau d'une hémi-langue associée à des dysesthésies. Ces symptômes étaient nocturnes et diurnes, soulagés à la mastication mais amplifiés à la fatigue ou lors de stress. Les deux patients ne présentaient pas de contexte psychologique particulier, n'avaient aucun antécédent médicaux ou chirurgicaux, ni aucun traitement pharmacologique. L'examen oral était normal.

Les symptômes du patient de 45 ans avaient débutés après une manipulation cervicale lors d'une séance d'ostéopathie. Une dysgueusie était apparue dans les jours suivants avant de laisser place à une sensation de brulure.

Chez les deux patients, un orthopantomogramme a permis de suspecter une styloïde ipsilatérale plus longue que la normale. La confirmation de cette anomalie a été obtenue par un examen tomodensitométrique qui a également permis d'éliminer tout processus tumoral. Cet examen montrait également une orientation différente de l'apophyse stylö̈de incriminée chez le patient ayant bénéficé d'ostéopathie.

Le diagnostic de syndrome d'Eagle a été obtenue par une régression temporaire des signes après infiltration locale au niveau du processus styloïde d'une solution de corticoïdes et bupivacaïne.

Le patient de 45 ans a bénéficié d'une résection chirurgicale de l'apophyse styloïde par voie transorale ce qui apermis la disparition des signes en l'espace de 2 mois. La patiente de 68 ans a refusé l'acte chirurgical. Un traitement médical à base d'amitriptylinea permis de diminuer les symptômes de $80 \%$.

This is an Open Access article distributed under the terms of the Creative Commons Attribution License 4.0, which permits unrestricted use, distribution, and reproduction in any medium, provided the original work is properly cited. 


\section{Discussion}

Cette forme rare du syndrome d'Eagle peut s'expliquer par des micro-traumatismes répétés de la chorde du tympan par l'apophyse stylö̈de anormalement longue ou déplacée (dans un des deux cas rapportés). En effet, il a été récemment suggéré que la chorde du tympan, qui participe à l'innervation de la langue, peut si elle est lésée provoquer des douleurs spontanées au niveau de l'hémilangue en lien avec une altération des contrôles inhibiteurs descendants de la douleur (Nasri-Hair 2011, Grushka 2003).

Ainsi, devant toute glossodynie atypique, il est raisonnable de poser l'hypothèse d'un syndrome de Eagle.

Nom et adresse du conférencier

\section{Christophe DESCHAUMES}

Service d'Odontologie - CHRU

1 place Raymond et Lucie Aubrac

63000 Clermont Ferrand Cedex (France)

christophe.deschaumes@udamail.fr 